\title{
Wilker and Huygens type inequalities for mixed trigonometric-hyperbolic functions
}

\author{
Yogesh J. Bagul ${ }^{1}$, Ramkrishna M. Dhaigude ${ }^{2}$, Barkat A. Bhayo*3, Vinay M. Raut ${ }^{4}$ \\ ${ }^{1}$ Department of Mathematics, \\ K. K. M. College, Manwath, \\ Dist: Parbhani(M. S.) - 431505, India \\ ${ }^{2}$ Department of Mathematics, \\ Government Vidarbha Institute of Science \\ and Humanities, Amravati(M. S.)-444604, India \\ *3 Department of Mathematics, Sukkur IBA University, \\ Sindh, Pakistan \\ ${ }^{4}$ Department of Mathematics, \\ Shri. Shivaji Science College, Amravati(M. S.)-444603, India
}

\begin{abstract}
Motivated by the work of Sándor [19], in this paper we establish a new Wilker type and Huygens type inequalities involving the trigonometric and hyperbolic functions. Moreover, in terms of hyperbolic functions, the upper and lower bounds of $\sin (x) / x$ and $\tan (x) / x$ are given.
\end{abstract}

Mathematics Subject Classification (2010). 26A09, 26D05, 26D20, 33B10

Keywords. Wilker-type inequality, Huygens-type inequality, trigonometric-hyperbolic functions.

\section{Introduction}

In the last two decades, the refinements of the inequalities involving trigonometric and hyperbolic functions such as Wilker type inequalities and Huygens type inequalities have been studied by several authors e.g., see $[4-7,10,17,19,22-24]$ and the references therein. Motivated by the work of Sándor [19], and above studies, in this paper we make a contribution to the subject by establishing a new Wilker type and Huygens type inequalities involving the trigonometric and hyperbolic functions. In all cases, we give the upper and lower bounds of $\sin (x) / x$ and $\tan (x) / x$ in terms of elementary functions.

For $0<x<\pi / 2$, Wilker [20] and Huygens [9] proposed the following inequalities

$$
\begin{gathered}
\left(\frac{\sin x}{x}\right)^{2}+\frac{\tan x}{x}>2, \\
2 \frac{\sin x}{x}+\frac{\tan x}{x}>3,
\end{gathered}
$$

respectively. In literature, inequality (1.1) and (1.2) are known as Wilker's and Huygens' inequalities, respectively.

In [24], Zhu proved the the hyperbolic version of (1.1) as follows,

$$
\left(\frac{\sinh x}{x}\right)^{2}+\frac{\tanh x}{x}>2, \quad x>0,
$$

\footnotetext{
*Corresponding Author.

Email addresses: yjbagul@gmail.com, rmdhaigude@gmail.com, barkat.bhayo@iba-suk.edu.pk, vinayraut18@gmail.com
} 
Similarly, Neuman and Sándor [16] established

$$
2 \frac{\sinh x}{x}+\frac{\tanh x}{x}>3 \quad x>0,
$$

as a hyperbolic version of (1.2).

Recently, $\mathrm{Wu}$ and Srivastava [21] proved that for $0<x<\pi / 2$

$$
\left(\frac{x}{\sin x}\right)^{2}+\frac{x}{\tan x}>2
$$

and the following hyperbolic version of (1.5)

$$
\left(\frac{x}{\sinh x}\right)^{2}+\frac{x}{\tanh x}>2 \quad(x>0)
$$

was proved by $\mathrm{Wu}$ and Debnath in [22].

For $0<x<\pi / 2$, the following relations were established by Neuman and Sándor

$$
\begin{gathered}
2 \frac{\sin x}{x}+\frac{\tan x}{x}>2 \frac{x}{\sin x}+\frac{x}{\tan x}>3, \\
2 \frac{\sinh x}{x}+\frac{\tanh x}{x}>2 \frac{x}{\sinh x}+\frac{x}{\tanh x}>3,
\end{gathered}
$$

in [16] and [17], respectively. For $0<x<\pi / 2$, one can write that

$$
\frac{\sin x}{x}+2 \frac{\tan x}{x}>2 \frac{\sin x}{x}+\frac{\tan x}{x},
$$

because $\cos x<1$.

For the extensions, generalizations, refinements and alternative proofs of the above inequalities, the reader is referred to see, e.g., $[4-7,11,12,14-18,21-25]$. Inequality (1.1) and (1.2) are known in the literature as Wilker's and Huygens inequalities respectively. Rest of the inequalities are known to be Wilker-type and Huygens-type inequalities for either trigonometric functions or hyperbolic functions. In contrast to this, Sándor [19] established the following inequalities for $0<x<\pi / 2$,

$$
\begin{gathered}
\left(\frac{\sinh x}{x}\right)^{2}+\frac{\sin x}{x}>2, \\
\left(\frac{x}{\sin x}\right)^{2}+\frac{x}{\sinh x}>2, \\
2 \frac{\sinh x}{x}+\frac{\sin x}{x}>3,
\end{gathered}
$$

and

$$
2 \frac{x}{\sin x}+\frac{x}{\sinh x}>3 .
$$

In [19], the inequalities (1.10), (1.11) are called as trigonometric-hyperbolic Wilker type inequalities and the inequalities (1.12), (1.13) are called as trigonometric-hyperbolic Huygens type inequalities. The main purpose of this paper is to obtain some trigonometrichyperbolic Wilker type and Huygens type inequalities.

The paper is organized as follows. In this section, we give an introduction and highlight the concerned previous inequalities together with the statements of main results in the form of theorems. Section 2 consists of preliminary ancestor and lemmas, which will be used in the proving procedures sequel. Section 3 closes the paper by proving the main results from Section 1.

Theorems 1.1-1.2 and Theorems 1.3-1.4 are dealing with Wilker-type inequalities and Huygens-type inequalities, respectively. 
Our first main result reads as follows.

Theorem 1.1. For $0<x<\pi / 2$ it is asserted that

$$
2-\frac{x^{2}}{2}<\frac{x}{\sin x}+\left(\frac{\tanh x}{x}\right)^{2}<2<2+\frac{x^{4}}{180}<\left(\frac{\sin x}{x}\right)^{2}+\frac{x}{\tanh x} .
$$

Theorem 1.2. The inequalities

$$
\frac{\sinh x}{x}+\left(\frac{x}{\tan x}\right)^{2}<2-\frac{x^{2}}{6}<2<2+\frac{x^{5}}{45 \tan x}<\left(\frac{\sinh x}{x}\right)^{2}+\frac{x}{\tan x}
$$

hold for all $x \in(0, \pi / 2)$.

Theorem 1.3. Let $0<x<\pi / 2$. Then the following inequalities hold:

$$
2 \frac{\sinh x}{x}+\frac{x}{\tan x}<3-\frac{x^{4}}{180}<3<3+\frac{31}{180} x^{4}<2 \frac{x}{\sinh x}+\frac{\tan x}{x}
$$

and

$$
\frac{\sinh x}{x}+2 \frac{x}{\tan x}<3-\frac{x^{2}}{2}<3<3+\frac{x^{2}}{2}<\frac{x}{\sinh x}+2 \frac{\tan x}{x} .
$$

Theorem 1.4. If $0<x<\pi / 2$ then the following inequalities hold:

$$
3-\frac{x^{4}}{180}<2 \frac{\sin x}{x}+\frac{x}{\tanh x}<3<2 \frac{x}{\sin x}+\frac{\tanh x}{x}<3+\frac{31}{180} x^{4},
$$

and

$$
3-\frac{x^{2}}{2}<\frac{x}{\sin x}+2 \frac{\tanh x}{x}<3<\frac{\sin x}{x}+2 \frac{x}{\tanh x}<3+\frac{x^{2}}{2} .
$$

\section{Preliminaries and Lemmas}

In this section, we give a few series expansion formulas and lemmas, which will be used in the proofs of our main result.

For the following series expansions, we refer to [8, 1.411].

$$
\begin{gathered}
\cos x=\sum_{k=0}^{\infty} \frac{(-1)^{k}}{(2 k) !} x^{2 k}, \quad \cosh x=\sum_{k=0}^{\infty} \frac{x^{2 k}}{(2 k) !}, \\
\frac{\sin x}{x}=\sum_{k=0}^{\infty} \frac{(-1)^{k}}{(2 k+1) !} x^{2 k}, \quad \frac{\sinh x}{x}=\sum_{k=0}^{\infty} \frac{x^{2 k}}{(2 k+1) !}, \quad x \neq 0, \\
\frac{x}{\sin x}=1+\sum_{k=1}^{\infty} \frac{2\left(2^{2 k-1}-1\right)}{(2 k) !}\left|B_{2 k}\right| x^{2 k}, \quad|x|<\pi, \\
\frac{x}{\sinh x}=1-\sum_{k=1}^{\infty} \frac{2\left(2^{2 k-1}-1\right)}{(2 k) !} B_{2 k} x^{2 k}, \quad|x|<\pi, \\
x \cot x=1-\sum_{k=1}^{\infty} \frac{2^{2 k}\left|B_{2 k}\right|}{(2 k) !} x^{2 k}, \quad|x|<\pi, \\
x \operatorname{coth} x=1+\sum_{k=1}^{\infty} \frac{2^{2 k} B_{2 k}}{(2 k) !} x^{2 k}, \quad|x|<\pi,
\end{gathered}
$$


Differentiating (2.8) we get

$$
\left.\begin{array}{rl}
\tan x & =\sum_{k=1}^{\infty} \frac{2^{2 k}\left(2^{2 k}-1\right)}{(2 k) !}\left|B_{2 k}\right| x^{2 k-1}, \quad|x|<\pi / 2 \\
& =x+\frac{x^{3}}{3}+\frac{2 x^{5}}{15}+\frac{17 x^{7}}{315}+\frac{62 x^{9}}{2835}+\cdots
\end{array}\right\}
$$

$$
\operatorname{sech}^{2} x=1-\tanh ^{2} x=\sum_{k=1}^{\infty} \frac{2^{2 k}(2 k-1)\left(2^{2 k}-1\right)}{(2 k) !} B_{2 k} x^{2 k-2}, \quad|x|<\pi / 2 .
$$

Hence

$$
\left(\frac{\tanh x}{x}\right)^{2}=-\sum_{k=2}^{\infty} \frac{2^{2 k}(2 k-1)\left(2^{2 k}-1\right)}{(2 k) !} B_{2 k} x^{2 k-4}, \quad|x|<\pi / 2 .
$$

The following lemmas are also necessary.

Lemma 2.1. [10] For $0<x<\pi / 2$, we have

$$
1-\frac{x^{2}}{6}<\frac{\sin x}{x}<\frac{x}{\sinh x} .
$$

Lemma 2.2. [4] For every $x \neq 0$, we have

$$
1+\frac{5 x^{2}}{x^{2}+15}<\frac{x}{\tanh x} .
$$

Lemma 2.3. For $0<x<\pi / 2$, it is true that

$$
\frac{x}{\tanh x}<\frac{\tan x}{x} .
$$

Proof. The famous Adamović-Mitrinović inequality [13]

$$
\cos x<\left(\frac{\sin x}{x}\right)^{3} \quad(0<x<\pi / 2)
$$

can be written as

$$
\left(\frac{x}{\sin x}\right)^{2}<\frac{\tan x}{x}(0<x<\pi / 2) .
$$

Combining this with the inequality [2, Proposition 1.2]

$$
\frac{x}{\tanh x}<\left(\frac{x}{\sin x}\right)^{2} \quad(0<x<\pi / 2),
$$

we get the required inequality.

Lemma 2.4. [1, $p$. 805]

$$
\frac{2(2 k) !}{(2 \pi)^{2 k}}<\left|B_{2 k}\right|<\frac{2(2 k) !}{(2 \pi)^{2 k}} \frac{2^{2 k-1}}{2^{2 k-1}-1}, \quad(k=1,2,3, \cdots)
$$

where $B_{2}, B_{4}, B_{6}, \cdots$ are Bernoulli numbers.

Lemma 2.5. The inequality

$$
\left|B_{2 k}\right|>\frac{1}{2^{2 k-1}(2 k+1)}=\frac{2^{1-2 k}}{(2 k+1)}
$$

holds for all integers $k \geq 2$. 
Proof. It is evident that

This yields

$$
\begin{gathered}
(2 k+1) !>\pi^{2 k} \quad(k=2,3, \cdots) \\
\text { i.e., } \frac{1}{\pi^{2 k}}>\frac{1}{(2 k+1) !} .
\end{gathered}
$$

$$
\left|B_{2 k}\right|>\frac{(2 k) !}{2^{2 k-1} \pi^{2 k}}>\frac{(2 k) !}{2^{2 k-1}(2 k+1) !}=\frac{2^{1-2 k}}{(2 k+1)}
$$

due to Lemma 2.4.

Lemma 2.6. The inequality

$$
\left|B_{2 k}\right|>\frac{1}{2^{2 k}(2 k+1)}=\frac{2^{-2 k}}{(2 k+1)}
$$

holds for all integers $k \geq 1$.

Proof. Since,

$$
2(2 k+1) !>\pi^{2 k} \quad(k=1,2,3, \cdots),
$$

we get the desired result by applying the same argument as in the proof of Lemma 2.5.

Lemma 2.7. The inequality

$$
\frac{\left|B_{2 k+2}\right|}{\left|B_{2 k}\right|}>\frac{(k+1)(2 k+1)\left(2^{2 k-1}-1\right)}{2^{2 k-1}\left(2^{2 k+2}-1\right)}
$$

is true for all integers $k \geq 2$.

Proof. From Lemma 2.4, we can write

$$
\begin{aligned}
\frac{\left|B_{2 k+2}\right|}{\left|B_{2 k}\right|} & >\frac{2(2 k+2) !}{(2 \pi)^{2 k+2}} \frac{(2 \pi)^{2 k}\left(2^{2 k-1}-1\right)}{2(2 k) ! 2^{2 k-1}} \\
& =\frac{(k+1)(2 k+1)\left(2^{2 k-1}-1\right)}{2^{2 k} \pi^{2}} \\
& >\frac{(k+1)(2 k+1)\left(2^{2 k-1}-1\right)}{2^{2 k-1}\left(2^{2 k+2}-1\right)}
\end{aligned}
$$

as $2^{2 k} \pi^{2}<2^{2 k-1}\left(2^{2 k+2}-1\right)$ i.e., $2 \pi^{2}+1<2^{2 k+2}$ for all integers $k \geq 2$.

\section{Proofs and corollaries}

Proof of Theorem 1.1. For the leftmost double inequality of (1.14), we add (2.3) and (2.9) to get

$$
\begin{aligned}
\frac{x}{\sin x}+\left(\frac{\tanh x}{x}\right)^{2} & =1+\sum_{k=1}^{\infty} \frac{2\left(2^{2 k-1}-1\right)}{(2 k) !}\left|B_{2 k}\right| x^{2 k} \\
& -\sum_{k=2}^{\infty} \frac{2^{2 k}(2 k-1)\left(2^{2 k}-1\right)}{(2 k) !} B_{2 k} x^{2 k-4}, \quad|x|<\pi / 2 \\
& =2+\sum_{k=1}^{\infty}\left[\frac{2\left(2^{2 k-1}-1\right)}{(2 k) !}\left|B_{2 k}\right|-\frac{2^{2 k+4}(2 k+3)\left(2^{2 k+4}-1\right)}{(2 k+4) !} B_{2 k+4}\right] x^{2 k} \\
& =2+\left(\sum_{k=1}^{\infty} a_{k} x^{2 k}:=A(x)\right), \quad|x|<\pi / 2
\end{aligned}
$$

where $a_{k}=\frac{2\left(2^{2 k-1}-1\right)}{(2 k) !}\left|B_{2 k}\right|-\frac{2^{2 k+4}(2 k+3)\left(2^{2 k+4}-1\right)}{(2 k+4) !} B_{2 k+4} \quad(k=1,2,3, \cdots)$. 
Clearly $a_{k}>0$ for $k=2,4,6, \cdots$. For $k=1,3,5,7, \cdots$ we claim that $a_{k}<0$ i.e.,

$$
\frac{\left|B_{2 k}\right|}{\left|B_{2 k+4}\right|}<\frac{2^{2 k+1}\left(2^{2 k+4}-1\right)}{(k+1)(k+2)(2 k+1)} \frac{1}{\left(2^{2 k-1}-1\right)}=L(k) .
$$

From Lemma 2.4, we write

$$
\left|B_{2 k}\right|<\frac{2(2 k) !}{2^{2 k} \pi^{2 k}} \frac{2^{2 k-1}}{\left(2^{2 k-1}-1\right)} \text { and } \frac{1}{\left|B_{2 k+4}\right|}<\frac{2^{2 k+4} \pi^{2 k+4}}{2(2 k+4) !} .
$$

Therefore

$$
\frac{\left|B_{2 k}\right|}{\left|B_{2 k+4}\right|}<\frac{1}{(k+1)(k+2)(2 k+1)} \frac{1}{\left(\left(2^{2 k-1}-1\right)\right.} \frac{\pi^{4} 2^{2 k+1}}{(2 k+3)}=M(k) .
$$

Since $\pi^{4}<(2 k+3)\left(2^{2 k+4}-1\right) \quad(k=1,2,3, \cdots)$ implies $M(k)<L(k)$, so $a_{k}<0$ for $k=1,3,5, \cdots$ and we conclude that $A(x)$ is an alternating convergent series whose first term is negative. The required double inequality follows by the truncation of $A(x)$.

For the rightmost double inequality of (1.14), we have by Lemmas 2.1 and 2.2 that

$$
\begin{aligned}
\left(\frac{\sin x}{x}\right)^{2}+\frac{x}{\tanh x} & >\left(1-\frac{x^{2}}{6}\right)^{2}+\left(1+\frac{5 x^{2}}{x^{2}+15}\right) \quad(0<x<\pi / 2) \\
& =2+\frac{x^{4}}{36}-\frac{x^{4}}{3 x^{2}+45} \\
& =2+\frac{x^{4}}{36}\left(\frac{x^{2}+3}{x^{2}+15}\right) \\
& >2+\frac{x^{4}}{36}\left(\frac{x^{2}+3}{5 x^{2}+15}\right)=2+\frac{x^{4}}{180}>2 .
\end{aligned}
$$

This completes the proof.

Remark 3.1. If $n$ is any odd positive integer and $0<x<\pi / 2$ then

$$
2+\sum_{k=1}^{n} a_{k} x^{2 k}<\frac{x}{\sin x}+\left(\frac{\tanh x}{x}\right)^{2}<2+\sum_{k=1}^{n+1} a_{k} x^{2 k}
$$

where $a_{k}$ is as defined in the proof of Theorem 1.1. In particular, we get

$$
2-\frac{x^{2}}{2}<\frac{x}{\sin x}+\left(\frac{\tanh x}{x}\right)^{2}<2-\frac{x^{2}}{2}+\frac{143 x^{4}}{360} .
$$

Proof of Theorem 1.2. As $\frac{x}{\tan x}<1 \quad(0<x<\pi / 2)$, we can write

$$
\frac{\sinh x}{x}+\left(\frac{x}{\tan x}\right)^{2}<\frac{\sinh x}{x}+\frac{x}{\tan x} \quad(0<x<\pi / 2) .
$$

Utilizing series expansions from (2.2) and (2.5),

$$
\begin{aligned}
\frac{\sinh x}{x}+\left(\frac{x}{\tan x}\right)^{2} & <\sum_{k=0}^{\infty} \frac{x^{2 k}}{(2 k+1) !}+1-\sum_{k=1}^{\infty} \frac{2^{2 k}\left|B_{2 k}\right|}{(2 k) !} x^{2 k} \\
& =2+\sum_{k=1}^{\infty} \frac{1}{(2 k) !}\left[\frac{1}{(2 k+1)}-2^{2 k}\left|B_{2 k}\right|\right] x^{2 k} \\
& =2+\left(\sum_{k=1}^{\infty} b_{k} x^{2 k}:=B(x)\right) \quad(0<x<\pi / 2),
\end{aligned}
$$


where $b_{k}=\frac{1}{(2 k) !}\left[\frac{1}{(2 k+1)}-2^{2 k}\left|B_{2 k}\right|\right]<0$ for all $k \geq 1$ by virtue of Lemma 2.6. So

$$
\frac{\sinh x}{x}+\left(\frac{x}{\tan x}\right)^{2}<2+b_{1} x^{2} \quad(0<x<\pi / 2)
$$

and by $b_{1}=-1 / 6$ we get the desired leftmost inequality of (1.15).

To prove the rightmost double inequality of (1.15), we equivalently prove that

$$
\tan x \sinh ^{2} x-2 x^{2} \tan x+x^{3}>\frac{x^{7}}{45}>0 \quad(0<x<\pi / 2) .
$$

Let

$$
f(x)=\tan x \sinh ^{2} x-2 x^{2} \tan x+x^{3} \quad(0<x<\pi / 2) .
$$

It can be written as

$$
f(x)=x^{3}-2 x^{2} \tan x+\frac{\tan x}{2} \cosh 2 x-\frac{\tan x}{2} .
$$

Using known series expansions of $\cosh x($ see $(2.1))$ and $\tan x$ (see (2.7)), we write

$$
\begin{aligned}
f(x) & =x^{3}-x^{2} \tan x+\sum_{k=2}^{\infty} \frac{2^{2 k-1}}{(2 k) !} x^{2 k} \tan x \\
& =x^{3}-x^{2}\left(x+\frac{1}{3} x^{3}+\frac{2}{15} x^{5}+\frac{17}{315} x^{7}+\frac{62}{2835} x^{9}+\cdots\right) \\
& +\frac{1}{3} x^{4} \tan x+\frac{2}{45} x^{6} \tan x+\frac{1}{315} x^{8} \tan x+\frac{2}{14175} x^{10} \tan x+\cdots \\
& =x^{3}+\left(-x^{3}-\frac{1}{3} x^{5}-\frac{2}{15} x^{7}-\frac{17}{315} x^{9}-\frac{62}{2835} x^{11}-\cdots\right) \\
& +\left(\frac{1}{3} x^{4}+\frac{2}{45} x^{6}+\frac{1}{315} x^{8}+\frac{2}{14175} x^{10}+\cdots\right) \times \\
& \left(x+\frac{1}{3} x^{3}+\frac{2}{15} x^{5}+\frac{17}{315} x^{7}+\frac{62}{2835} x^{9}+\cdots\right) \\
& =\left(-\frac{1}{3} x^{5}-\frac{2}{15} x^{7}-\frac{17}{315} x^{9}-\frac{62}{2835} x^{11}-\cdots\right) \\
& +\left(\frac{1}{3} x^{5}+\frac{7}{45} x^{7}+\frac{59}{945} x^{9}+\frac{2492}{99225} x^{11}+\cdots\right) \\
& =\frac{1}{45} x^{7}+\frac{8}{945} x^{9}+\frac{46}{14175} x^{11}+\cdots>\frac{1}{45} x^{7}>0 .
\end{aligned}
$$

The proof is completed.

Remark 3.2. The rightmost inequality of (1.15) can be put as

$$
\left(\frac{\sinh x}{x}\right)^{2}+\frac{x}{\tan x}\left(1-\frac{x^{4}}{45}\right)>2 \quad(0<x<\pi / 2) .
$$

Corollary 3.3. For $0<x<\pi / 2$, we have the following chains of inequalities:

$$
\left(\frac{x}{\sin x}\right)^{2}+\frac{\tanh x}{x}>\left(\frac{\sinh x}{x}\right)^{2}+\frac{\tanh x}{x}>\left(\frac{\sinh x}{x}\right)^{2}+\frac{x}{\tan x}>2+\frac{x^{5}}{45 \tan x}>2
$$

and

$$
\left(\frac{x}{\sinh x}\right)^{2}+\frac{\tan x}{x}>\left(\frac{\sin x}{x}\right)^{2}+\frac{\tan x}{x}>\left(\frac{\sin x}{x}\right)^{2}+\frac{x}{\tanh x}>2 .
$$

Proof. The chain of inequalities in (3.1) immediately follows by applying Theorem 1.2 with Lemmas 2.1 and 2.3. Similarly (3.2) follows due to Theorem 1.1 and Lemmas 2.1 and 2.3. 
Again the following corollary is an easy consequence of Theorems 1.1 and 1.2.

Corollary 3.4. For $0<x<\pi / 2$, we have following chain of inequalities:

$$
\left(\frac{x}{\sin x}\right)^{2}+\frac{x}{\tan x}>\left(\frac{\sinh x}{x}\right)^{2}+\frac{x}{\tan x}>2+\frac{x^{5}}{45 \tan x}>2
$$

and

$$
\left(\frac{x}{\sinh x}\right)^{2}+\frac{x}{\tanh x}>\left(\frac{\sin x}{x}\right)^{2}+\frac{x}{\tanh x}>2 .
$$

As the applications of Theorems 1.1 and 1.2, we have in fact obtained/refined the inequalities (1.1)-(1.6) in Corollaries 3.3 and 3.4.

Proof of Theorem 1.3. We first prove the inequalities in (1.16). For the leftmost inequality, we have by (2.2) and (2.5) that

$$
\begin{aligned}
2 \frac{\sinh x}{x}+\frac{x}{\tan x} & =\sum_{k=0}^{\infty} \frac{2}{(2 k+1) !} x^{2 k}+1-\sum_{k=1}^{\infty} \frac{2^{2 k}\left|B_{2 k}\right|}{(2 k) !} x^{2 k} \\
& =3+\sum_{k=1}^{\infty} \frac{2}{(2 k+1) !} x^{2 k}-\sum_{k=1}^{\infty} \frac{2^{2 k}\left|B_{2 k}\right|}{(2 k) !} x^{2 k} \\
& =3+\sum_{k=2}^{\infty} \frac{2}{(2 k) !}\left(\frac{1}{2 k+1}-2^{2 k-1}\left|B_{2 k}\right|\right) x^{2 k} \\
& <3-\frac{x^{4}}{180}<3
\end{aligned}
$$

by Lemma 2.5 .

For the rightmost inequality of (1.16) we use (2.4) and (2.7) to get

$$
\begin{aligned}
2 \frac{x}{\sinh x}+\frac{\tan x}{x} & =2-\sum_{k=1}^{\infty} \frac{2^{2}\left(2^{2 k-1}-1\right)}{(2 k) !} B_{2 k} x^{2 k}+\sum_{k=1}^{\infty} \frac{2^{2 k}\left(2^{2 k}-1\right)}{(2 k) !}\left|B_{2 k}\right| x^{2 k-2} \\
& =2-\sum_{k=1}^{\infty} \frac{2^{2}\left(2^{2 k-1}-1\right)}{(2 k) !} B_{2 k} x^{2 k}+\sum_{k=0}^{\infty} \frac{2^{2 k+2}\left(2^{2 k+2}-1\right)}{(2 k+2) !}\left|B_{2 k+2}\right| x^{2 k} \\
& =3+\sum_{k=2}^{\infty} \frac{2^{2}}{(2 k) !}\left[\frac{2^{2 k-1}\left(2^{2 k+2}-1\right)}{(k+1)(2 k+1)}\left|B_{2 k+2}\right|-(-1)^{k+1}\left(2^{2 k-1}-1\right)\left|B_{2 k}\right|\right] x^{2 k} \\
& =3+\left(\sum_{k=2}^{\infty} c_{k} x^{2 k}:=C(x)\right),
\end{aligned}
$$

where $c_{k}=\frac{2^{2}}{(2 k) !}\left[\frac{2^{2 k-1}\left(2^{2 k+2}-1\right)}{(k+1)(2 k+1)}\left|B_{2 k+2}\right|-(-1)^{k+1}\left(2^{2 k-1}-1\right)\left|B_{2 k}\right|\right] \quad(k \geq 2)$. Now $c_{k}>0$ for $k=2,4,6, \cdots$ and

$$
c_{k}=\frac{2^{2}}{(2 k) !}\left[\frac{2^{2 k-1}\left(2^{2 k+2}-1\right)}{(k+1)(2 k+1)}\left|B_{2 k+2}\right|-\left(2^{2 k-1}-1\right)\left|B_{2 k}\right|\right]>0 \text { for } k=3,5,7, \cdots
$$

by Lemma 2.7. Thus all the terms of $C(x)$ are positive and hence by truncating $C(x)$ we get

$$
2 \frac{x}{\sinh x}+\frac{\tan x}{x}>3+c_{2} x^{4}>3 \text { where } c_{2}=\frac{31}{180} .
$$

The inequalities (1.17) can be proved easily by applying similar arguments. 
Proof of Theorem 1.4. We give proof of the inequalities in (1.18) only. The inequalities in (1.19) will follow similarly. From (2.2) and (2.6) we have

$$
\begin{aligned}
2 \frac{\sin x}{x}+\frac{x}{\tanh x} & =2 \sum_{k=0}^{\infty} \frac{(-1)^{k}}{(2 k+1) !} x^{2 k}+1+\sum_{k=1}^{\infty} \frac{2^{2 k} B_{2 k}}{(2 k) !} x^{2 k} \\
& =3+\sum_{k=1}^{\infty} \frac{2(-1)^{k}}{(2 k+1) !} x^{2 k}+\sum_{k=1}^{\infty} \frac{2^{2 k}\left|B_{2 k}\right|}{(2 k) !} x^{2 k} \\
& =3+\sum_{k=1}^{\infty} \frac{2}{(2 k) !}\left[\frac{(-1)^{k}}{2 k+1}-(-1)^{k} 2^{2 k-1}\left|B_{2 k}\right|\right] x^{2 k} \\
& =3+\sum_{k=2}^{\infty} \frac{2(-1)^{k}}{(2 k) !}\left[\frac{1}{2 k+1}-2^{2 k-1}\left|B_{2 k}\right|\right] x^{2 k} \\
& =3+\left(\sum_{k=2}^{\infty} d_{k} x^{2 k}:=D(x)\right)
\end{aligned}
$$

where $d_{k}=\frac{2(-1)^{k}}{(2 k) !}\left[\frac{1}{2 k+1}-2^{2 k-1}\left|B_{2 k}\right|\right] \quad(k \geq 2)$. By Lemma 2.5 , the series $D(x)$ is an alternating convergent series and its first term is negative. Consequently

$$
3+d_{2} x^{4}<2 \frac{\sin x}{x}+\frac{x}{\tanh x}<3
$$

where $d_{2}=-\frac{1}{180}$. This gives leftmost double inequality of (1.18).

For the rightmost double inequality of (1.18), we make use of (2.3) and (2.8) to obtain

$$
\begin{aligned}
2 \frac{x}{\sin x}+\frac{\tanh x}{x} & =2+\sum_{k=1}^{\infty} \frac{2^{2}\left(2^{2 k-1}-1\right)}{(2 k) !}\left|B_{2 k}\right| x^{2 k}+\sum_{k=1}^{\infty} \frac{2^{2 k}\left(2^{2 k}-1\right)}{(2 k) !} B_{2 k} x^{2 k-2} \\
& =2+\sum_{k=1}^{\infty} \frac{2^{2}\left(2^{2 k-1}-1\right)}{(2 k) !}\left|B_{2 k}\right| x^{2 k}+\sum_{k=1}^{\infty} \frac{2^{2 k+2}\left(2^{2 k+2}-1\right)}{(2 k+2) !} B_{2 k+2} x^{2 k} \\
& =3+\sum_{k=2}^{\infty} \frac{2^{2}}{(2 k) !}\left[\left(2^{2 k-1}-1\right)\left|B_{2 k}\right|+(-1)^{k} \frac{2^{2 k-1}\left(2^{2 k+2}-1\right)}{(k+1)(2 k+1)}\left|B_{2 k+2}\right|\right] x^{2 k} \\
& =3+\left(\sum_{k=2}^{\infty} e_{k} x^{2 k}=E(x)\right),
\end{aligned}
$$

where $e_{k}=\frac{2^{2}}{(2 k) !}\left[\left(2^{2 k-1}-1\right)\left|B_{2 k}\right|+(-1)^{k} \frac{2^{2 k-1}\left(2^{2 k+2}-1\right)}{(k+1)(2 k+1)}\left|B_{2 k+2}\right|\right] \quad(k \geq 2)$. Clearly $e_{k}>0$ for $k=2,4,6, \cdots$ and $e_{k}<0$ for $k=3,5,7, \cdots$ by Lemma 2.7. Thus $E(x)$ is an alternating convergent series whose first term is positive, i.e. $\frac{31}{180} x^{4}$. The desired inequality follows by the truncation of $E(x)$. This ends the proof.

Remark 3.5. If $n$ is even positive integer and $0<x<\pi / 2$ then

$$
3+\sum_{k=2}^{n} d_{k} x^{2 k}<2 \frac{\sin x}{x}+\frac{x}{\tanh x}<3+\sum_{k=2}^{n+1} d_{k} x^{2 k},
$$

and

$$
3+\sum_{k=2}^{n+1} e_{k} x^{2 k}<2 \frac{x}{\sin x}+\frac{\tanh x}{x}<3+\sum_{k=2}^{n} e_{k} x^{2 k},
$$

where $d_{k}$ and $e_{k}$ are as defined in the proof of Theorem 1.4.

Before concluding this section, we must emphasize that the Huygens-type inequalities in Theorems 1.3 and 1.4 give sharp bounds to the much-discussed functions in the literature 
such as $\sin (x) / x, x / \tan (x)$ etc. For example, the inequalities

$$
\frac{\sin x}{x}<\frac{1}{2}\left(3-\frac{x}{\tanh x}\right) \quad(0<x<\pi / 2),
$$

and

$$
\frac{\sinh x}{x}<\frac{1}{2}\left(3-\frac{x}{\tan x}\right) \quad(0<x<\pi / 2)
$$

are very sharp and interesting for further studies.

\section{References}

[1] M. Abramowitz and I. A. Stegun, eds., Handbook of Mathematical Functions with Formulas, Graphs, and Mathematical Tables, Dover-New York, 1965.

[2] Y. J. Bagul, C. Chesneau, Some new simple inequalities involving exponential, trigonometric and hyperbolic functions, Cubo. A Mathematical Journal 21(1) (2019), pp. 21-35. Doi: 10.4067/S0719-06462019000100021

[3] Y. J. Bagul, C. Chesneau, Two double sided inequalities involving sinc and hyperbolic sinc functions, Int. J. Open Problems Compt. Math., Vol. 12, No. 4, pp. 15-20, 2019.

[4] G. Bercu, and S. Wu, Refinements of certain hyperbolic inequalities via the Padé approximation method, J. Nonlinear Sci. Appl., Vol. 9, No. 7, pp. 5011-5020, 2016. Doi: $10.22436 /$ jnsa.009.07.05

[5] B. A. Bhayo, J. Sándor, On Jordan's, Redheffer's and Wilker's inequality, Math. Inequal. Appl., Vol. 19, No. 3, pp. 823-839, 2016. Doi: 10.7153/mia-19-60

[6] H.-H. Chu, Z.-H. Yang, Y.-M. Chu, and W. Zhang, Generalized Wilker-type inequalities with two parameters, J. Inequal. Appl., 2016:187, 2016. Doi: 10.1186/s13660-016$1127-8$

[7] B.-N. Guo, B.-M. Qiao, F. Qi, and W. Li, On new proofs of Wilker's inequalities involving trigonometric functions, Math. Inequal. Appl., Vol. 6, No. 1, pp. 19-22, 2003.

[8] I. S. Gradshteyn and I. M. Ryzhik, Table of Integrals, Series and Products, Elsevier, edn. 2007.

[9] C. Huygens, Oeuvres Completes, Société Hollandaise des Sciences, Haga, 1888-1940.

[10] R. Klén, M. Visuri, and M. Vuorinen, On Jordan type inequalities for hyperbolic functions, J. Inequal. Appl., Vol. 2010, Article ID 362548, 14 pages, 2010. Doi: $10.1155 / 2010 / 362548$

[11] L. Matejíčka, Note on two new Wilker-type inequalities, Int. J. Open Problems Compt. Math., Vol. 4, No. 1, pp. 79-85, 2011.

[12] A. Mhanna, On a general Huygens-Wilker inequality, Applied Mathematics E-Notes, Vol. 20, pp. 79-81, 2020.

[13] D. S. Mitrinović, Analytic Inequalities, Springer-Verlag, Berlin, (1970).

[14] C. Mortici, A subtly analysis of Wilker inequality, Appl. Math. Comput., Vol. 231, pp. 516-520, 2014. Doi: 10.1016/j.amc.2014.01.017

[15] K. Nantomah, An alternative proof of an inequality by Zhu, International Journal of Mathematical Analysis, Vol. 14, No. 3, pp. 133-136, 2020. Doi: 10.12988/ijma.2020.91292

[16] E. Neuman, and J. Sándor, On some inequalities involving trigonometric and hyperbolic functions with emphasis on the Cusa-Huygens, Wilker, and Huygens inequalities, Math. Inequal. Appl., Vol. 13, No. 4, pp. 715-723, 2010. Doi: 10.7153/mia-13-50.

[17] E. Neuman, On Wilker and Huygens type inequalities, Math. Inequal. Appl., Vol. 15, No. 2, pp. 271-279, 2012. Doi: 10.7153/mia-15-22

[18] M. Rašajski, T. Lutovac, B. Malešević, Sharpening and Generalizations of ShaferFink and Wilker type inequalities: a new approach, J. Nonlinear Sci. Appl., Vol. 11, No. 7, pp. 885-893, 2018. Doi: 10.22436/jnsa.011.07.02 
[19] J. Sándor, On some new Wilker and Huygens type trigonometric-hyperbolic inequalities, Proceedings of the Jangjeon Mathematical Society, Vol. 15, No. 2, pp. 145-153, 2012.

[20] J. B. Wilker, Elementary Problems: E3301-E3306, Amer. Math. Monthly, Vol. 96, No. 1, pp. 54-55, 1989.

[21] S.-H. Wu, and H. M. Srivastava, A weighted and exponential generalization of Wilker's inequality and its applications, Integral Transforms Spec. Funct., Vol. 18, No. 7-8, pp. 529-535, 2007. Doi: 10.1080/10652460701284164

[22] S. Wu, and L. Debnath, Wilker-type inequalities for hyperbolic functions, Appl. Math. Lett., Vol. 25, No. 5, pp. 837-842, 2012. Doi: 10.1016/j.aml.2011.10.028

[23] B. Zhang, and C.-P. Chen, Sharp Wilker and Huygens type inequalities for trigonometric and inverse trigonometric functions, J. Math. Inequal., Vol. 14, No. 3, pp. 673-684, 2020. Doi: 10.7153/jmi-2020-14-43

[24] L. Zhu, On Wilker-type inequalities, Math. Inequal. Appl., Volume 10, No. 4, pp. 727-731, 2007. Doi: 10.7153/mia-10-67

[25] L. Zhu, New inequalities of Wilker's type for circular functions, AIMS Mathematics, Vol. 5, No. 5, pp. 4874-4888, 2020. Doi: 10.3934/math.2020311 